\title{
From the Galaxy to the Edge of the Universe: Plausible Sources of UHECRs
}

\author{
Angela V. Olinto \\ Department of Astronomy \&3 Astrophysics, \\ 8 Enrico Fermi Institute, \\ The University of Chicago, Chicago, IL 60637
}

\begin{abstract}
The lack of a high energy cutoff in the cosmic ray spectrum together with an apparently isotropic distribution of arrival directions for the highest energy events have strongly constrained most models proposed for the generation of these particles. An overview of the theoretical proposals are presented along with their most general signatures. Future experimental tests of the different proposals are discussed.
\end{abstract}

\section{INTRODUCTION}

The surprising detection of cosmic rays with energies above $10^{20} \mathrm{eV}$ has triggered considerable interest on the origin and nature of these particles. In addition to the ultra-high energy events detected by Fly's Eye, Haverah Park, Yakutsk, and Volcano Ranch, the AGASA experiment has recently reported many hundreds of events (728) accumulated with energies above $10^{19} \mathrm{eV}$ and 8 events above $10^{20} \mathrm{eV}$ [1]. (For a recent review of the observations, see, e.g., [2].)

These observations are surprising because not only the energy requirements for astrophysical sources to accelerate particles to $>10^{20} \mathrm{eV}$ are extraordinary, but the propagation of particles at these energies is prone to large energy losses. Reactions of ultra-high energy proton, nuclei, or photon primaries with cosmic background radiation in intergalactic space suppress the observable flux at the highest energies significantly. In fact, cosmic ray protons of energies above a few $10^{19} \mathrm{eV}$ reach the $\Delta$ resonance threshold and produce pions off the cosmic microwave background (CMB) limiting their source to lie not much further than about $50 \mathrm{Mpc}$ away from Earth. This photopion production gives rise to the well-known Greisen-ZatsepinKuzmin (GZK) cutoff in the spectrum of cosmic ray photons [3]. Nuclei are photodisintegrated on shorter distances due to the infrared background [4] while the radio background constrains photons to originate from even closer systems [5].

If these UHE particles are protons, they likely originate in extragalactic sources, since at these high energies the Galactic magnetic field cannot confine protons in the Galaxy. If protons are extragalactic, they traverse large intergalactic distances 
so their spectrum should exhibit the GZK cutoff. The shape of the GZK cutoff depends on the source input spectrum and the distribution of sources in space as well as in the intergalactic magnetic field. In Figure 1, we contrast the observed flux by AGASA with the expected flux for proton sources distributed homogeneously or distributed like galaxies with injection spectrum $J(E) \propto E^{-\gamma}$ and $\gamma=3[6,7]$. We model the distribution of UHECR sources by using the galaxy distribution measured by the recent IRAS redshift survey know as PSCz [8]. As can be seen from the figure, even allowing for the local overdensity the observations are consistently above the theoretical expectation. In fact, when we normalize our simulations by requiring that the number of events with $E \geq 10^{19} \mathrm{eV}$ equals the AGASA observations (728), we find that the number of expected events for $E \geq 10^{20} \mathrm{eV}$ is only $1.2 \pm 1.0$ for the PSCz case, i.e., $6 \sigma$ away from the observed 8 events.

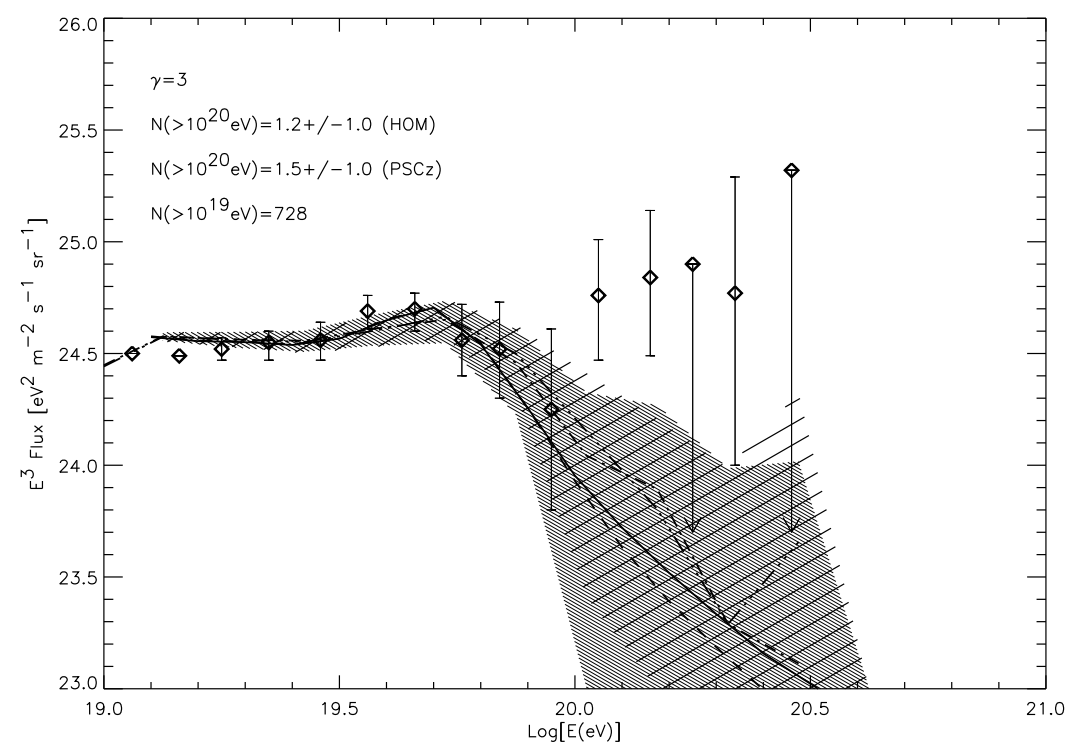

FIGURE 1. Simulated fluxes for the AGASA statistics of 728 events above $10^{19} \mathrm{eV}$, and $\gamma=3$, using a homogeneous source distribution $\backslash$ hatches) and the PSCz distribution (dense / hatches). The solid and dashed lines are the results of the analytical calculations for the same two cases. The dash-dotted and dash-dot-dot-dotted lines trace the mean simulated fluxes for the homogeneous and the PSCz cases. (see [7]).

The gap between observed flux and model predictions narrows as the injection spectrum of the ultra-high energy cosmic ray (UHECR) sources becomes much harder than $\gamma=3$. The results for $\gamma=2.1$ are shown in Figure 2. For $\gamma=2.1$, the number of expected events above $10^{20} \mathrm{eV}$ reaches $3.3 \pm 1.6$ for a homogeneous distribution while for the PSCz catalog it is $3.7 \pm 2.0$. This trend can be seen also in Figure 3, where analytic solutions for $\gamma=1.5,2.1$ and 2.7 are shown.

In addition to the presence of events past the GZK cutoff, there has been no clear counterparts identified in the arrival direction of the highest energy events. If these 


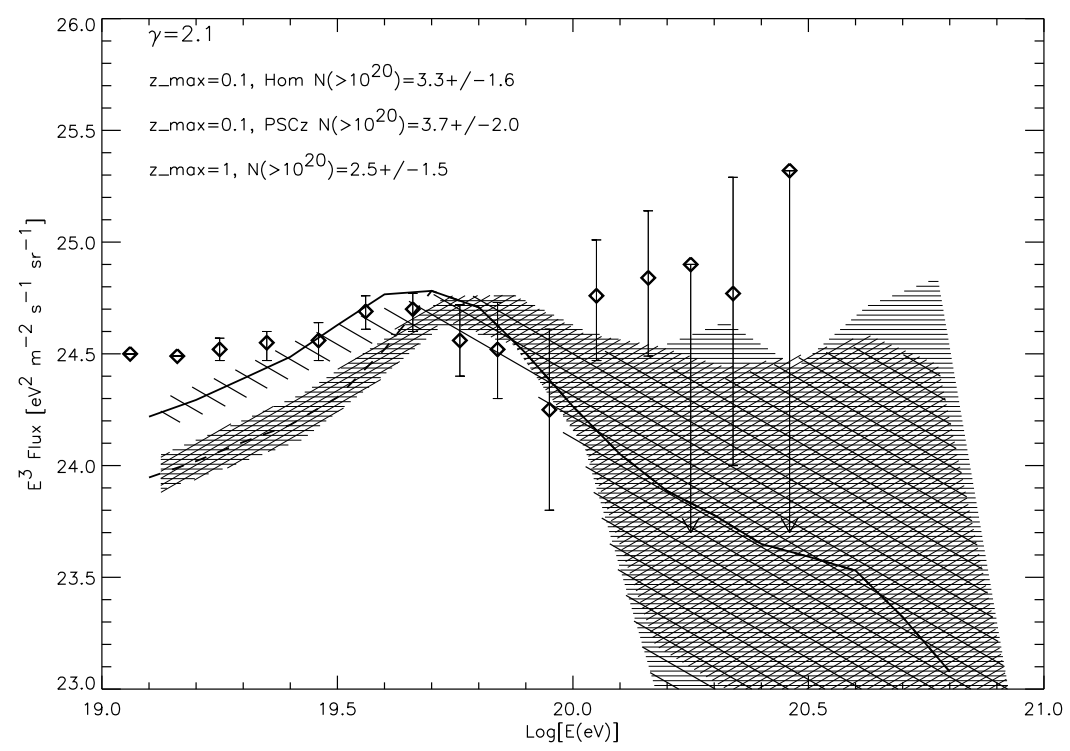

FIGURE 2. Simulated fluxes for the AGASA statistics of 728 events above $10^{19} \mathrm{eV}$, and $\gamma=2.1$, using a homogeneous source distribution with $z_{\max }=0.1$ (/ hatches), the PSCz distribution with $z_{\max }=0.1$ (horizontal hatches), and a homogeneous source distribution with $z_{\max }=1 \wedge$ hatches).

events are protons or photons, these observations should be astronomical, i.e., their arrival directions should be the angular position of sources. At these high energies the Galactic and extragalactic magnetic fields do not affect proton orbits significantly so that even protons should point back to their sources within a few degrees. Protons at $10^{20} \mathrm{eV}$ propagate mainly in straight lines as they traverse the Galaxy since their gyroradii are $\sim 100 \mathrm{kpc}$ in $\mu \mathrm{G}$ fields which is typical in the Galactic disk. Extragalactic fields are expected to be $\ll \mu \mathrm{G}[9,10]$, and induce at most $\sim$ $1^{\circ}$ deviation from the source. Even if the Local Supercluster has relatively strong fields, the highest energy events may deviate at most $\sim 10^{\circ}[11,12]$. At present, no correlations between arrival directions and plausible optical counterparts such as sources in the Galactic plane, the Local Group, or the Local Supercluster have been clearly identified. Ultra high energy cosmic ray data are consistent with an isotropic distribution of sources in sharp contrast to the anisotropic distribution of light within $50 \mathrm{Mpc}$ from Earth.

The absence of a GZK cutoff and the isotropy of arrival directions are two of the many challenges that models for the origin of UHECRs face. This is an exciting open field, with many scenarios being proposed but no clear front runner. Not only the origin of these particles may be due to physics beyond the standard model of particle physics, but their existence can be used to constrain extensions of the standard model such as violations of Lorentz invariance (see, e.g., [13]).

In the next section, we summarize the astrophysical Zevatron proposals while 
models that involve new physics are discussed in the following section. To conclude, future observational tests of UHECR models and their implications are discussed. (For recent reviews see [14-17].)

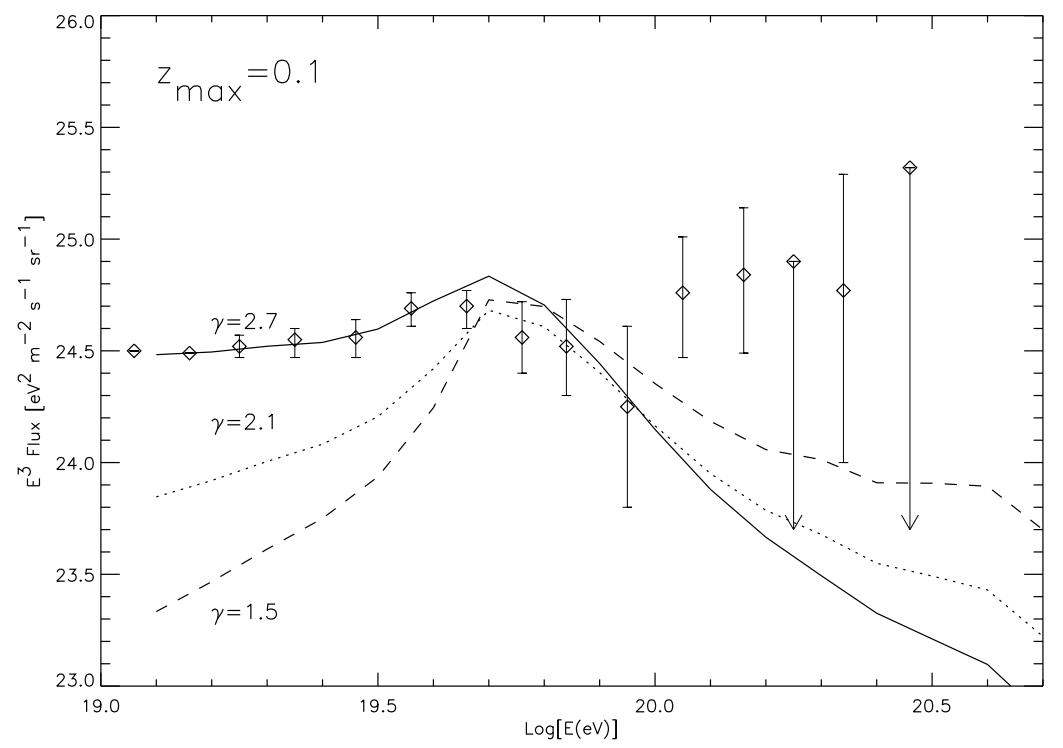

FIGURE 3. Propagated spectrum for source spectral index of $\gamma=1.5,2.1,2.7$

\section{ASTROPHYSICAL ZEVATRONS}

The puzzle presented by the observations of cosmic rays above $10^{20} \mathrm{eV}$ have generated a number of proposals that we divide here as Astrophysical Zevatrons and New Physics models. Astrophysical Zevatrons are also referred to as bottom-up models and involve searching for acceleration sites in known astrophysical objects that can reach ZeV energies. New Physics proposals can be either hybrid or pure top-down models. Hybrid models involve Zevatrons and extensions of the particle physics standard model while top-down models involve the decay of very high mass relics from the early universe and physics way beyond the standard model. Here we discuss astrophysical Zevatrons while new physics models are discussed in the next section.

Cosmic rays can be accelerated in astrophysical plasmas when large-scale macroscopic motions, such as shocks, winds, and turbulent flows, are transferred to individual particles. The maximum energy of accelerated particles, $E_{\max }$, can be estimated by requiring that the gyroradius of the particle be contained in the acceleration region: $E_{\max }=Z e B L$, where $Z e$ is the charge of the particle, $B$ is the strength and $L$ the coherence length of the magnetic field embedded in the plasma. For $E_{\text {max }} \gtrsim 10^{20} \mathrm{eV}$ and $Z \sim 1$, the only known astrophysical sources with reason- 
able $B L$ products are neutron stars $\left(B \sim 10^{13} \mathrm{G}, L \sim 10 \mathrm{~km}\right)$, active galactic nuclei $(\mathrm{AGNs})\left(B \sim 10^{4} \mathrm{G}, L \sim 10 \mathrm{AU}\right)$, radio lobes of $\mathrm{AGNs}(B \sim 0.1 \mu \mathrm{G}, L \sim 10 \mathrm{kpc})$, and clusters of galaxies $(B \sim \mu \mathrm{G}, L \sim 100 \mathrm{kpc})$. In Figure 4 , we highlight the $B$ vs. $L$ for objects that can reach $E_{\max }=10^{20} \mathrm{eV}$ with $Z=1$ (dashed line) and $Z=26$ (solid line). We discuss each of these candidates below.

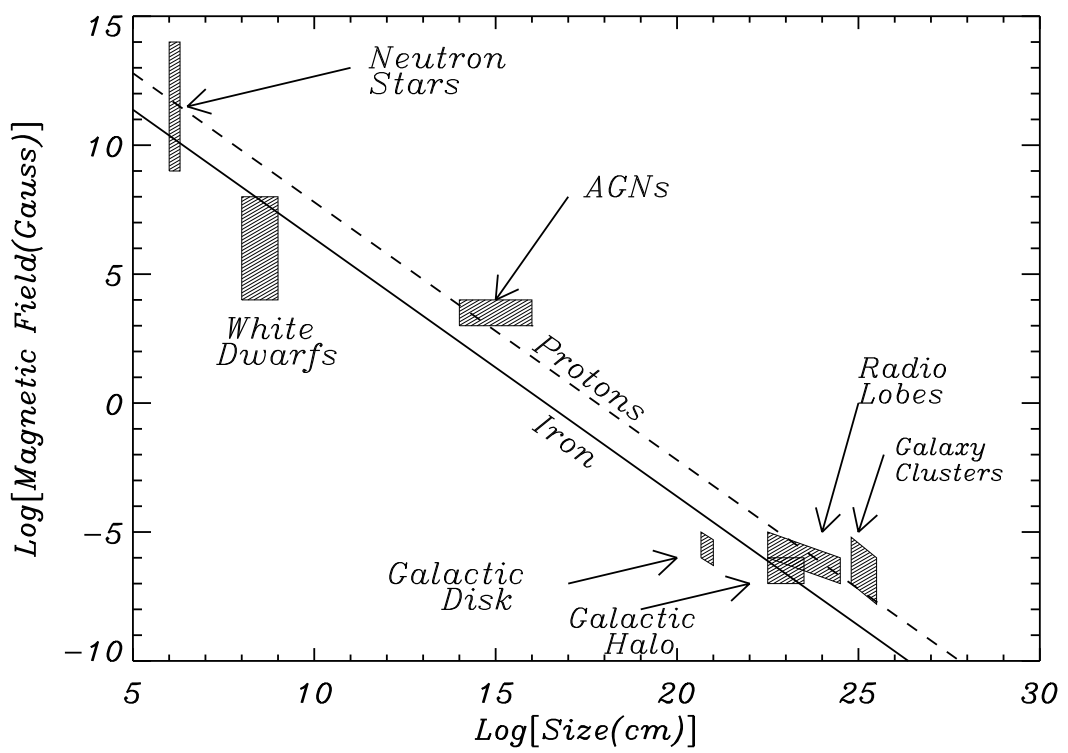

FIGURE 4. $B$ vs. $L$, for $E_{\max }=10^{20} \mathrm{eV}, Z=1$ (dashed line) and $Z=26$ (solid line) from [14].

Clusters of Galaxies: Cluster shocks are reasonable sites to consider for ultrahigh energy cosmic ray (UHECR) acceleration, since particles with energy up to $E_{\max }$ can be contained by cluster fields. However, efficient losses due to photopion production off the CMB during the propagation inside the cluster limit UHECRs in cluster shocks to reach at most $\sim 10 \mathrm{EeV}[18]$.

AGN Radio Lobes: Next on the list of plausible Zevatrons are extremely powerful radio galaxies [19]. Jets from the central black-hole of an active galaxy end at a termination shock where the interaction of the jet with the intergalactic medium forms radio lobes and 'hot spots'. Of special interest are the most powerful AGNs where shocks can accelerate particles to energies well above an $\mathrm{EeV}$ via the firstorder Fermi mechanism. These sources may be responsible for the flux of UHECRs up to the GZK cutoff [20].

A nearby specially powerful source may be able to reach energies past the cutoff. However, extremely powerful AGNs with radio lobes and hot spots are rare and far apart. The closest known object is M87 in the Virgo cluster ( $\sim 18 \mathrm{Mpc}$ away) and could be a main source of UHECRs. Although a single nearby source with especially hard spectra may fit the spectrum for a given strength and structure of the intergalactic magnetic field [21], it is unlikely to match the observed arrival 
direction distribution. If M87 is the primary source of UHECRs a concentration of events in the direction of M87 or the Virgo cluster should be seen in the arrival direction distribution. No such hot spot is observed (Hot spot in Table 1). The next known nearby source after M87 is NGC315 which is already too far at a distance of 80 Mpc. Any unknown source between M87 and NGC315 would likely contribute a second hot spot, not a isotropic distribution. The very distant radio lobes will contribute a GZK cut spectrum (dist RLs in Table 1).

The lack of a clear hot spot in the direction of M87 from the arrival direction distribution has encouraged the idea that a strong Galactic magnetic wind may exist that could help isotropize the arrival directions of UHECRs. A Galactic wind with a strongly magnetized azimuthal component [22] ( $B_{G W}$ in Table 1) can significantly alter the paths of UHECRs such that the observed arrival directions of events above $10^{20} \mathrm{eV}$ would trace back to the North Galactic pole which is close to the Virgo where M87 resides. If our Galaxy has a such a wind is yet to be determined. The proposed wind would focus most observed events into the northern Galactic pole and render point source identification fruitless [23]. Future observations of UHECRs from the Southern Hemisphere by the Southern Auger Site will provide precious data on previously unobserved parts of the sky and help distinguish plausible proposals for the effect of local magnetic fields on arrival directions. Full sky coverage is a key discriminator of such proposals.

AGN - Central Regions: The powerful engines that give rise to the observed jets and radio lobes are located in the central regions of active galaxies and are powered by the accretion of matter onto supermassive black holes. It is reasonable to consider the central engines themselves as the likely accelerators $[24, ?]$. In principle, the nuclei of generic active galaxies (not only the ones with radio lobes) can accelerate particles via a unipolar inductor not unlike the one operating in pulsars. In the case of AGNs, the magnetic field ( $B_{\text {source }}$ in Table 1$)$ may be provided by the infalling matter and the spinning black hole horizon provides the imperfect conductor for the unipolar induction.

The problem with AGNs as UHECR sources is two-fold: first, UHE particles face debilitating losses in the acceleration region due to the intense radiation field present in AGNs, and second, the spatial distribution of objects should give rise to a GZK cutoff of the observed spectrum. In the central regions of AGNs, loss processes are expected to downgrade particle energies well below the maximum achievable energy. This limitation has led to the proposal that quasar remnants, supermassive black holes in centers of inactive galaxies, are more effective UHECR accelerators [25]. In this case, losses at the source are not as significant but the propagation from source to us should still lead to a clear GZK cutoff since sources would be associated with the large scale structure of the galaxy distribution ( $L S S$ in Table 1). From Figure 1-3, these models can only succeed if the source spectrum is fairly hard $(\gamma \lesssim 2)[7]$.

Neutron Stars: Another astrophysical system capable of accelerating UHECRs is a neutron star. In addition to having the ability to confine $10^{20} \mathrm{eV}$ protons (Figure 4 ), the rotation energy of young neutron stars is more than sufficient to match the 
observed UHECR fluxes [26]. However, acceleration processes inside the neutron star light cylinder are bound to fail much like the AGN central region case: ambient magnetic and radiation fields induce significant losses. However, the plasma that expands beyond the light cylinder is free from the main loss processes and may be accelerated to ultra high energies.

One possible source of UHECR past the GZK cutoff is the early evolution of neutron stars. In particular, newly formed, rapidly rotating neutron stars may accelerate iron nuclei to UHEs through relativistic MHD winds beyond their light cylinders [27]. This mechanism naturally leads to vary hard injection spectra $(\gamma \simeq 1)$ (see Table 1). As seen in Figure 3, $\gamma \sim 1$ improves the agreement between predicted flux and observations for energies above $10^{20} \mathrm{eV}$. In this case, UHECRs originate mostly in the Galaxy and the arrival directions require that the primaries be heavier nuclei. Depending on the structure of Galactic magnetic fields, the trajectories of iron nuclei from Galactic neutron stars may be consistent with the observed arrival directions of the highest energy events [28]. Moreover, if cosmic rays of a few times $10^{18} \mathrm{eV}$ are protons of Galactic origin, the isotropic distribution observed at these energies is indicative of the diffusive effect of the Galactic magnetic fields on iron at $\sim 10^{20} \mathrm{eV}$. This proposal should be constrained once the primary composition is clearly determined (see Iron in Table 1).

It has also been suggested that young extragalactic highly magnetized neutron stars (magnetars) may be sources of UHE protons which are accelerated by reconnection events [29]. These would be prone to a GZK cut spectrum and would need a very hard injection spectrum to become viable explanations.

Gamma-Ray Bursts: Transient high energy phenomena such as gamma-ray bursts (GRBs) may also be a source of ultra-high energies protons [30]. In addition to both phenomena having unknown origins, GRBs and UHECRs have other similarities that may argue for a common source. Like UHECRs, GRBs are distributed isotropically in the sky, and the average rate of $\gamma$-ray energy emitted by GRBs is comparable to the energy generation rate of UHECRs of energy $>10^{19} \mathrm{eV}$ in a redshift independent cosmological distribution of sources, both have $\approx 10^{44} \mathrm{erg} / \mathrm{Mpc}^{3} / \mathrm{yr}$.

However, recent GRB counterpart identifications argue for a strong cosmological evolution for GRBs. The redshift dependence of GRB distribution is such that the flux of UHECR associated with nearby GRBs would be too small to fit the UHECR observations [31]. In addition, the distribution of UHECR arrival directions and arrival times argues against the GRB-UHECR common origin. Events past the

TABLE 1. ZEVATRONS

\begin{tabular}{lccccc}
\hline Zevatron & Composition & Source $\gamma$ & Sky Distrib. & $B$ Needs & Best Tests \\
\hline Radio Lobes & Proton & $2-3$ & M87 +dist RLs & $B_{G W}$ & Hot spot \& $\gamma$ \\
AGN Center & Proton & $2-3$ & LSS & $B_{\text {source }}$ & GZK feature \\
YNSWs & Iron & 1 & Gal. Disk & $B_{\text {gal }}$ & Iron \& Disk \\
GRBs & Proton & $2-3$ & Hot spot or & large $B_{I G M}$ & Hot spot \& Flux \\
\hline
\end{tabular}


GZK cutoff require that only GRBs from $\lesssim 50 \mathrm{Mpc}$ contribute. Since less than about one burst is expected to have occurred within this region over a period of $100 \mathrm{yr}$, the unique source would appear as a concentration of UHECR events in a small part of the sky (a Hot spot in Table 1). In addition, the signal would be very narrow in energy $\Delta E / E \sim 1$. Again, a strong intergalactic magnetic field can ease the some of these difficulties giving a very large dispersion in the arrival time and direction of protons produced in a single burst (large $B_{I G M}$ in Table 1) [30]. Finally, if the observed small scale clustering of arrival directions is confirmed by future experiments with clusters having some lower energy events clearly precede higher energy ones, bursts would be invalidated [32].

\section{NEW PHYSICS MODELS}

The UHECR puzzle has inspired a number of different models that involve physics beyond the standard model of particle physics. New Physics proposals can be topdown models or a hybrid of astrophysical Zevatrons with new particles. Top-down models involve the decay of very high mass relics that could have been formed in the early universe.

The most economical among hybrid proposals involves a familiar extension of the standard model, namely, neutrino masses. If some flavor of neutrinos have mass (e.g., $\sim 0.1 \mathrm{eV}$ ), the relic neutrino background is a target for extremely high energy neutrinos to interact and generate other particles by forming a Z-boson that subsequently decays [33] (see $\nu Z$ burst in Table 2). If the universe has very luminous sources (Zevatrons) of extremely high energy neutrinos $\left(\gg 10^{21} \mathrm{eV}\right)$, these neutrinos would traverse very large distances before annihilating with neutrinos in the smooth cosmic neutrino background. The UHE neutrino Zevatrons can be much further than the GZK limited volume, since neutrinos do not suffer the GZK losses. But if the interaction occurs throughout a large volume, the GZK feature should also be observed. For plausible neutrino masses $\sim 0.1 \mathrm{eV}$, the neutrino background is very unclustered, so the arrival direction for events should be isotropic and small scale clustering may be a strong challenge for this proposal. The weakest link in this proposal is the nature of a Zevatron powerful enough to accelerate protons above tens of $\mathrm{ZeVs}$ that can produce $\mathrm{ZeV}$ neutrinos as secondaries. This Zevatron is quite spectacular, requiring an energy generation in excess of presently known highest energy sources (referred to as $\nu$ Zevatron in Table 2).

Another suggestion is that the UHECR primary is a new hadronic particle that is also accelerated in Zevatrons. The mass of a hypothetical hadronic primary can be limited by the shower development of the Fly's Eye highest energy event to be below $\lesssim 50 \mathrm{GeV}$ [34]. As in the Z-burst proposal, a neutral particle is usually harder to accelerate and are usually created as secondaries of even higher energy charged primaries. But once formed these can traverse large distances without being affected by cosmic magnetic fields. Thus, a signature for future experiments of hybrid models that invoke new particles as primaries is a clear correlation between 
the position of powerful Zevatrons in the sky such as distant compact radio quasars and the arrival direction of UHE events [35]. Preliminary evidence for such a correlation has been recently reported [36].

Another exotic primary that can be accelerated to ultra high energies by astrophysical systems is the vorton. Vortons are small loops of superconducting cosmic string stabilized by the angular momentum of charge carriers [37]. Vortons can be a component of the dark matter in galactic halos and be accelerated by astrophysical magnetic fields [38]. Vortons as primaries can be constrained by the observed shower development profile.

It is possible that none of the astrophysical scenarios or the hybrid new physics models are able to explain present and future UHECR data. In that case, the alternative is to consider top-down models. Top-down models involve the decay of monopole-antimonoploe pairs [39], ordinary and superconducting cosmic strings, cosmic necklaces, vortons, and superheavy long-lived relic particles. The idea behind these models is that relics of the very early universe, topological defects (TDs) or superheavy relic (SHR) particles, produced after or at the end of inflation, can decay today and generate UHECRs. Defects, such as cosmic strings, domain walls, and magnetic monopoles, can be generated through the Kibble mechanism as symmetries are broken with the expansion and cooling of the universe. Topologically stable defects can survive to the present and decompose into their constituent fields as they collapse, annihilate, or reach critical current in the case of superconducting cosmic strings. The decay products, superheavy gauge and higgs bosons, decay into jets of hadrons, mostly pions. Pions in the jets subsequently decay into $\gamma$-rays, electrons, and neutrinos. Only a few percent of the hadrons are expected to be nucleons. Typical features of these scenarios are a predominant release of $\gamma$-rays and neutrinos and a QCD fragmentation spectrum which is considerably harder than the case of Zevatron shock acceleration.

$\mathrm{ZeV}$ energies are not a challenge for top-down models since symmetry breaking scales at the end of inflation typically are $\gg 10^{21} \mathrm{eV}$ (typical X-particle masses vary between $\sim 10^{22-25} \mathrm{eV}$ ). Fitting the observed flux of UHECRs is the real challenge since the typical distances between TDs is the Horizon scale about several Gpc. The low flux hurts proposals based on ordinary and superconducting cosmic strings which are distributed throughout space (Distant TD in Table 2). Monopoles usually suffer the opposite problem, they would in general be too numerous. Inflation succeeds in diluting the number density of monopoles and makes them too rare for UHECR production. To reach the observed UHECR flux, monopole models usually involve some degree of fine tuning. If enough monopoles and antimonopoles survive from the early universe, they may form a bound state, named monopolonium, that can decay generating UHECRs. The lifetime of monopolonia may be too short for this scenario to succeed unless they are connected by strings [40].

Once two symmetry breaking scales are invoked, a combination of horizon scales gives room to reasonable number densities. This can be arranged for cosmic strings that end in monopoles making a monopole string network or even more clearly for cosmic necklaces [41]. Cosmic necklaces are hybrid defects where each monopole is 


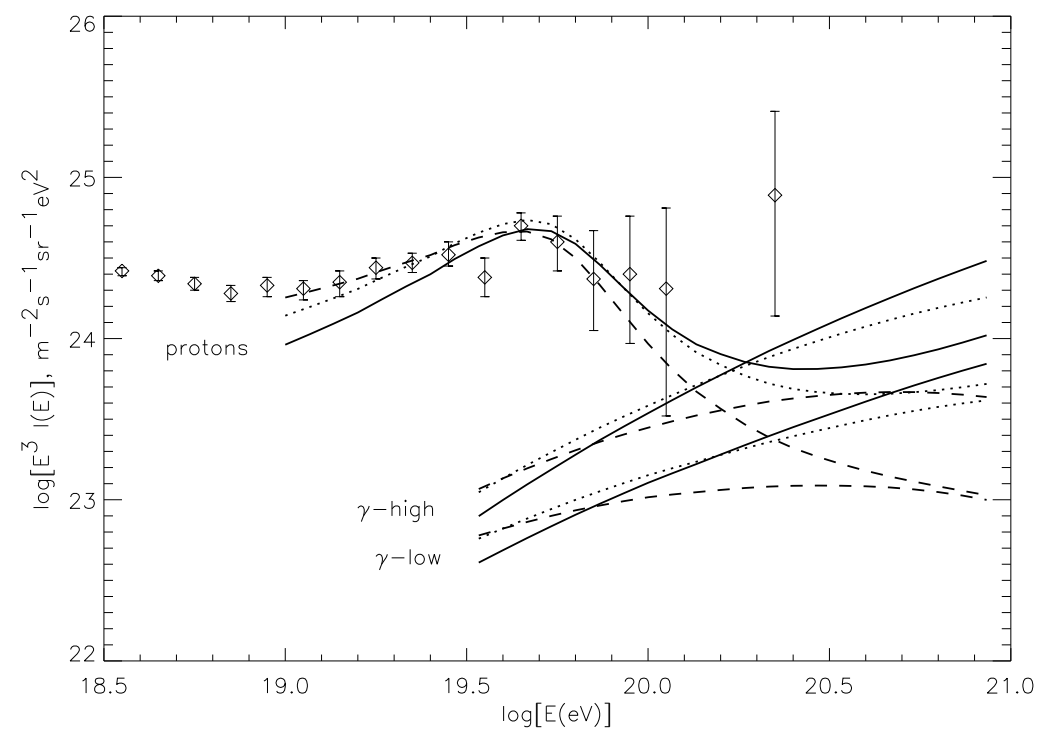

FIGURE 5. Proton and $\gamma$-ray fluxes from necklaces for $m_{X}=10^{14} \mathrm{GeV}$ (dashed lines), $10^{15}$ $\mathrm{GeV}$ (dotted lines), and $10^{16} \mathrm{GeV}$ (solid lines) normalized to the observed data. $\gamma$-high and $\gamma$-low correspond to two extreme cases of $\gamma$-ray absorption (see, [35]).

connected to two strings resembling beads on a cosmic string necklace. Necklace networks may evolve to configurations that can fit the UHECR flux which is ultimately generated by the annihilation of monopoles with antimonopoles trapped in the string $[41,42]$. In these scenarios, protons dominate the flux in the lower energy side of the GZK cutoff while photons tend to dominate at higher energies depending on the radio background (see Figure 5 and Distant TD in Table 2). If future data can settle the composition of UHECRs from 0.01 to $1 \mathrm{ZeV}$, these models can be well constrained. In addition to fitting the UHECR flux, topological defect models are constrained by limits on the flux of high energy photons, from $10 \mathrm{MeV}$ to 100 GeV, observed by EGRET.

Another interesting possibility is the recent proposal that UHECRs are produced by the decay of unstable superheavy relics that live much longer than the age of the universe [43]. SHRs may be produced at the end of inflation by non-thermal effects such as a varying gravitational field, parametric resonances during preheating, instant preheating, or the decay of topological defects. These models need to invoke special symmetries to insure unusually long lifetimes for SHRs and that a sufficiently small percentage decays today producing UHECRs [43,44]. As in the topological defects case, the decay of these relics also generates jets of hadrons. These particles behave like cold dark matter and could constitute a fair fraction of the halo of our Galaxy. Therefore, their halo decay products would not be limited by the GZK cutoff allowing for a large flux at UHEs (see Figure 6 and SHRs in Table 2). Similar signatures can occur if topological defects are microscopic, such 


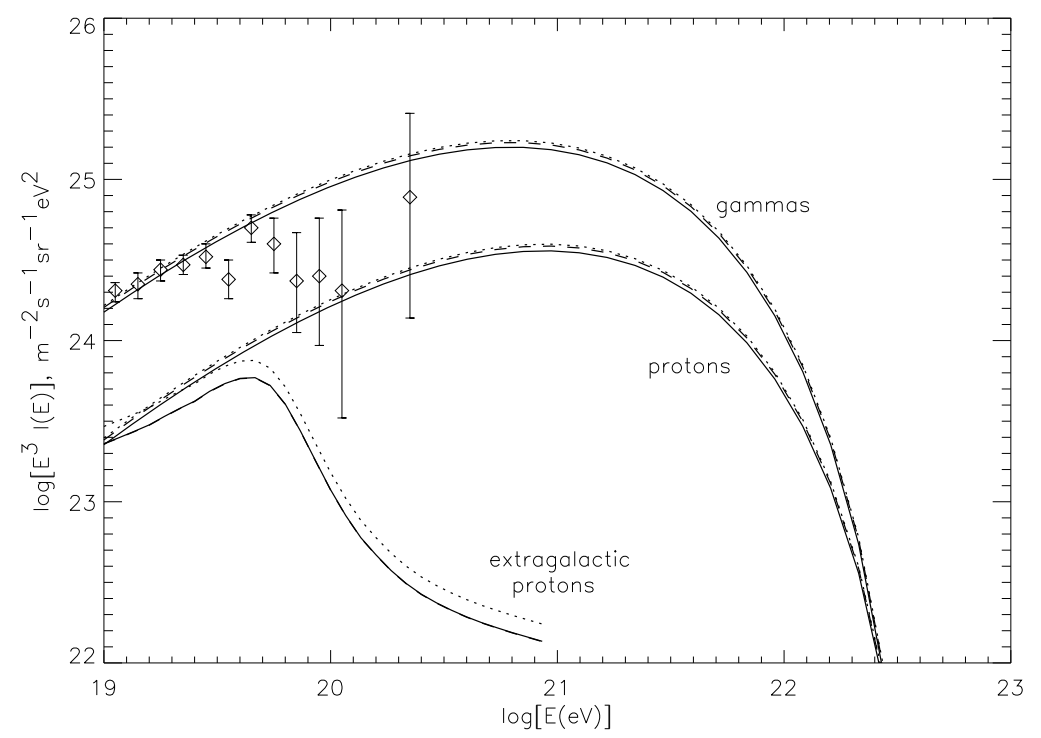

FIGURE 6. SHRs or monopolia decay fluxes (for $m_{X}=10^{14} \mathrm{GeV}$ ): nucleons from the halo (protons), $\gamma$-rays from the halo (gammas) and extragalactic protons. Solid, dotted and dashed curves correspond to different model parameters (see [35]).

as monopolonia and vortons, and decay in the Halo of our Galaxy (Local TD in Table 2). In both cases (SHRs and Local TD) the composition of the primary would be a good discriminant since the decay products are usually dominated by photons.

Future experiments should be able to probe these hypotheses. For instance, in the case of SHR and monopolonium decays, the arrival direction distribution should be close to isotropic but show an asymmetry due to the position of the Earth in the Galactic Halo [42] and the clustering due to small scale dark matter inhomogeneities $[6,45]$. Studying plausible halo models for their expected asymmetry and inhomogeneitis will help constrain halo distributions especially when larger data sets are available in the future. High energy gamma ray experiments such as GLAST will also help constrain SHR models via the electromagnetic decay products [46].

TABLE 2. NEW PHYSICS

\begin{tabular}{lccccc}
\hline Source & Composition & Source $\gamma$ & Sky Distrib. & Th. Challenge & Best Tests \\
\hline$\nu$ Z burst & photons & $\nu$ Zevatron & Isotropic & $\nu$ Zevatron & photon, Isotropy \\
Distant TD & phot/GZK p & QCD frag & Isotropic & flux & photon, GZK p \\
Local TD & photons & QCD frag & Gal Halo & origin & photon, Halo \\
SHRs & photons & QCD frag & Gal Halo & lifetime & photon, Halo \\
\hline
\end{tabular}




\section{CONCLUSION}

Next generation experiments such as the High Resolution Fly's Eye which recently started operating, the Pierre Auger Project which is now under construction, the proposed Telescope Array, and the EUSO and OWL satellites will significantly improve the data at the extremely-high end of the cosmic ray spectrum [2]. With these observatories a clear determination of the spectrum and spatial distribution of UHECR sources is within reach.

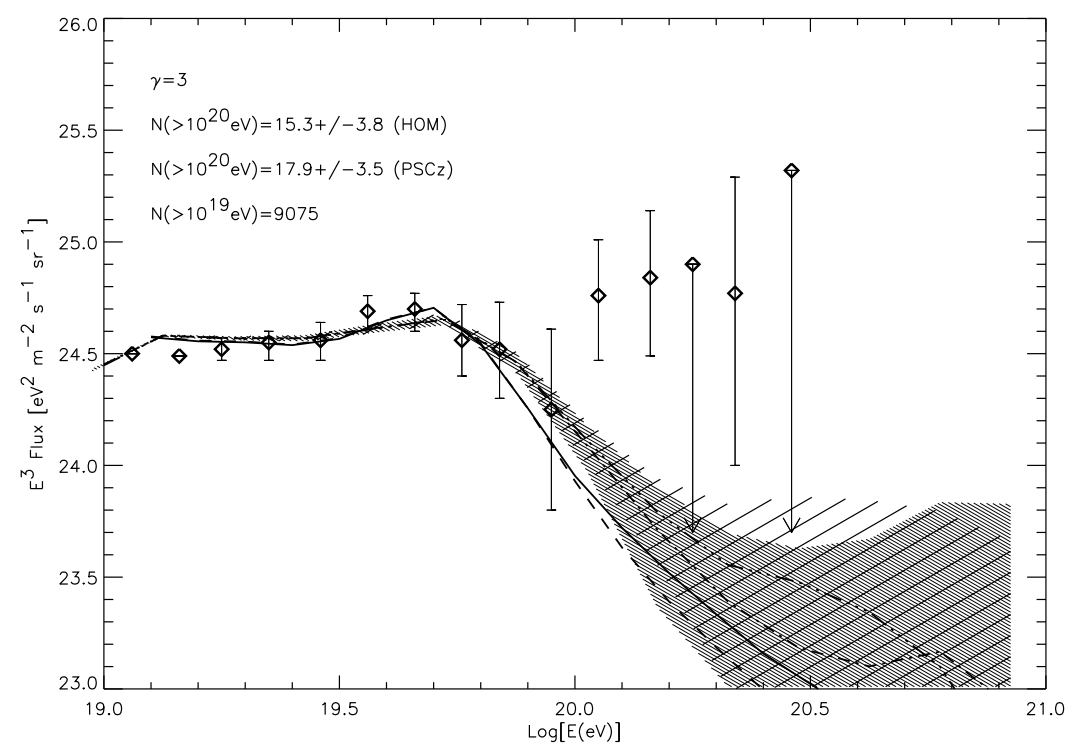

FIGURE 7. Simulated fluxes for the Auger projected statistics of 9075 events above $10^{19} \mathrm{eV}$, and $\gamma=3$, using a homogeneous source distribution $\backslash$ hatches) and the PSCz distribution (/ hatches). The solid and dashed lines are the results of the analytical calculations for the same two cases. The dash-dotted and dash-dot-dot-dotted lines trace the mean simulated fluxes for the homogeneous and the PSCz cases. (see [7]).

The lack of a GZK cutoff should become clear with HiRes and Auger and most extragalactic Zevatrons may be ruled out. The observed spectrum will distinguish Zevatrons from new physics models by testing the hardness of the spectrum and the effect of propagation. Figure 7 shows how clearly Auger will test the spectrum in spite of clustering properties. The cosmography of sources should also become clear and able to discriminate between plausible populations for UHECR sources. The correlation of arrival directions for events with energies above $10^{20} \mathrm{eV}$ with some known structure such as the Galaxy, the Galactic halo, the Local Group or the Local Supercluster would be key in differentiating between different models. For instance, a correlation with the Galactic center and disk should become apparent at extremely high energies for the case of young neutron star winds, while a correlation with the large scale galaxy distribution should become clear for the 
case of quasar remnants. If SHRs or monopolonia are responsible for UHECR production, the arrival directions should correlate with the dark matter distribution and show the halo asymmetry. For these signatures to be tested, full sky coverage is essential. Finally, an excellent discriminator would be an unambiguous composition determination of the primaries. In general, Galactic disk models invoke iron nuclei to be consistent with the isotropic distribution, extragalactic Zevatrons tend to favor proton primaries, while photon primaries are more common for early universe relics. The hybrid detector of the Auger Project should help determine the composition by measuring the depth of shower maximum and the muon content of the same shower. The prospect of testing extremely high energy physics as well as solving the UHECR mystery awaits improved observations that should be coming in the next decade with experiments under construction such as Auger [47] or in the planning stages such as the Telescope Array [48], EUSO [49], and OWL [50].

\section{ACKNOWLEDGMENT}

Many thanks to the organizers of this excellent workshop. This work was supported by NSF through grant AST-0071235 and DOE grant DE-FG0291 ER40606.

\section{REFERENCES}

1. N. Hayashida et al., astro-ph/0008102, appendix for Astrophys. J. 522 (1999) 225.

2. A. A. Watson, Phys. Rept. 333-334 (2000) 309; X. Bertou, M. Boratav, and A. Letessier-Selvon, Int.J.Mod.Phys. A15 (2000) 2181-2224.

3. K. Greisen, Phys. Rev. Lett. 16 (1966) 748; G. T. Zatsepin and V. A. Kuzmin, Sov. Phys. JETP Lett. 4 (1966) 78.

4. J. L. Puget, F. W. Stecker, and J. H. Bredekamp, Astrophys. J. 205 (1976) 638; F. W. Stecker and M. H. Salamon, Astrophys. J. 512 (1999) 521.

5. V. S. Berezinsky, Yad. Fiz. 11 (1970) 339; R. J. Protheroe and P. L. Biermann, Astropart. Phys. 6 (1996) 45; erratum ibid. 7 (1997) 181.

6. P. Blasi, in this volume.

7. M. Blanton, P. Blasi, \& A. V. Olinto, astro-ph/0009466.

8. W. Saunders et al., (astro-ph/0001117).

9. P. P. Kronberg, Rep. Prog. Phys., 57 (1994) 325; J. P. Vallée, Fundamentals of Cosmic Physics, 19 (1997) 1.

10. P. Blasi, S. Burles, and A. V. Olinto, Astrophys. J. 514 (1999) L79.

11. D. Ryu, H. Kang and P. L. Bierman, Astron. Astrophys. 335 (1998) 19.

12. G. Sigl, M. Lemoine, and P. Biermann, Astropart. Phys. 10 (1999) 141.

13. L. Gonzalez-Mestres, Nucl. Phys. B (Proc. Suppl.) 48 (1996) 131; S. Coleman and S. L. Glashow, Phys. Rev. D 59 (1999) 116008; R. Aloisio, P. Blasi, P Ghia, and A. Grillo, preprint INFN/AE-99/24.

14. A. V. Olinto, Phys. Rept. 333-334 (2000) 329.

15. P. Bhattacharjee and G. Sigl, Phys. Rept. 327 (2000) 109. 
16. V. S. Berezinsky, Nucl.Phys. (Proc.Suppl.) 70 (1999) 41.

17. R. D. Blandford, Particle Physics and the Universe, eds. Bergstrom, Carlson and Fransson (World Scientific, 1999).

18. H. Kang, D. Ryu, T.W. Jones, Astropart. Phys. 456 (1996) 422.

19. P.L. Biermann and P. Strittmatter, Astropart. Phys. 322 (1987) 643.

20. J. P. Rachen and P. L. Biermann, Astron. Astrophys. 272 (1993) 161.

21. P. Blasi and A. V. Olinto, Phys. Rev. D 59 (1999) 023001.

22. E. J. Ahn, G. Medina-Tanco, P. Biermann, T. Stanev, astro-ph/9911123 (1999).

23. P. Billoir and A Letessier-Selvon, astroph/000142 (2000).

24. K.S. Thorne, R. Price, \& D. MacDonals, Black Holes: The Membrane Paradigm (New Haven: Yale Press) (1986).

25. E. Boldt and P. Ghosh, Mon. Not. R. Astron. Soc., in press (1999).

26. A. Venkatesan, M. C. Miller, and A. V. Olinto, Astrophys. J. 484 (1997) 323.

27. A. V. Olinto, R. I. Epstein, and P. Blasi, Proceedings of 26th ICRC, Salt Lake City, 4, 361 (1999); P. Blasi, R. I. Epstein, and A. V. Olinto, Astrophys. J. Letters 533 (2000) L123.

28. V. N. Zirakashvili, et al., Astron. Lett. 24 (1998) 139.

29. E.M. de Gouveia Dal Pino, A. Lazarian, Astrophys. J. 536 (2000) L31-L34.

30. E. Waxman, Phys. Rev. Lett. 75 (1995) 386; M. Vietri, Ap. J. 453 (1995) 883.

31. F. W. Stecker, Astropart. Phys. 14 (2000) 207.

32. G. Sigl, M. Lemoine, and A. V. Olinto, Phys. Rev. D 56 (1997) 4470.

33. D. Fargion, B. Mele, and A. Salis, Astrophys.J. 517 (1999) 725-733; T. Weiler, Astropar. Phys. 11 (1999) 303.

34. I. F. Albuquerque, G. Farrar, and E. Kolb, Phys. Rev. D 59 (1999) 015021.

35. G. R. Farrar and P. L. Biermann, Phys. Rev. Lett. 81 (1998) 3579.

36. A. Virmani, et al., astro-ph/0010235

37. R. L. Davis and E. P. S. Shellard, Nucl. Phys. B 323 (1989) 209.

38. S. Bonazzola and P. Peter, Astropart. Phys. 7, 161 (1997).

39. C. T. Hill, Nucl. Phys. B 224 (1983) 469; D. N. Schramm and C.T. Hill, Proc. 18th ICRC (Bangalore) 2 (1983) 393.

40. J. J. Blanco-Pillado and K. D. Olum, Phys.Rev. D60 (1999) 083001.

41. V. Berezinsky and A. Vilenkin, Phys. Rev. Lett. 79 (1997) 5202.

42. V. Berezinsky, P. Blasi, and A. Vilenkin, Phys. Rev. D 58 (1998) 103515-1.

43. V. Berezinsky, M. Kachelrieß and A. Vilenkin, Phys. Rev. Lett. 79 (1997) 4302; V. Kuzmin and V. Rubakov, Yad. Fisika 61 (1998) 1122.

44. D. J. H. Chung, E. W. Kolb, and A. Riotto, Phys. Rev. Lett. 81 (1998) 4048; V. Kuzmin and I. Tkachev, Phys. Rev. D 59 (1999) 123006.

45. P. Blasi \& R. K. Seth, Phys. Lett. B 486 (2000) 233-238.

46. P. Blasi, Phys. Rev. D 60 (1999) 023514.

47. J. W. Cronin, Nucl. Phys. B. (Proc. Suppl.) 28 (1992) 213.

48. M. Teshima et al., Nucl. Phys. B (Proc. Suppl.) 28B (1992) 169.

49. L. Scarsi, in this volume.

50. R. E. Streitmatter, Proc. of Workshop on Observing Giant Cosmic Ray Air Showers from $>10^{20}$ eV Particles from Space, eds. J. F. Krizmanic, J. F. Ormes, and R. E. Streitmatter (AIP Conference Proceedings 433, 1997). 